CIC. Cuadernos de Información y Comunicación ISSN: 1135-7991

http://dx.doi.org/10.5209/ciyc.64842

\title{
Activismo y narrativas gamificadas: estudio comparativo de entornos ciberdemocráticos de empoderamiento ciudadano
}

\author{
Marta Pérez-Escolar ${ }^{1}$; Pablo Navazo Ostúa ${ }^{1}$
}

Recibido: 25/04/2019 /Aceptado: 1/06/2019

Resumen. El objetivo principal de este estudio se centra en conocer si las narrativas gamificadas pueden representar en una valiosa herramienta de empoderamiento ciudadano con la que motivar la participación de individuo en entornos ciberdemocráticos. Con este propósito, se ha seleccionado un conjunto representativo de dos productos gamificados de corte activista: el documental interactivo "Fort McMoney" y el serious game "A Force More Powerful". Para ello, se ha recurrido a una metodología cualitativa donde se hibrida el modelo Octalysis (Chou, 2015) y el paradigma de la Escalera de participación (Arnstein, 1969). Los resultados muestran que las narrativas gamificadas son un instrumento de empoderamiento ciudadano que sirven para favorecer acciones ciberactivistas. Sin embargo, estos proyectos pueden confluir también en un pseudoactivismo para vagos.

Palabras clave: Participación ciudadana, ciberdemocracia, narrativas gamificadas, juego, empoderamiento ciudadano, activismo; gamificación.

\section{[en] Activism and gamification narratives: comparative study of cyber- democratic environments for citizen empowerment}

\begin{abstract}
The main objective of this study is focused on understanding whether gamification narratives can represent a useful tool for citizen empowerment with which to motivate individual participation in cyber-democratic environments. Regarding this purpose, a representative group of two gamification products with an activist undertone have been selected: the interactive documentary "Fort McMoney" and the serous game "A Force More Powerful". Then, this research has applied a qualitative methodology that combines the Octalysis model (Chou, 2015) and the Ladder of participation paradigm (Arnstein, 1969). The results show that gamification narratives are a tool for citizen empowerment and favour cyber-activist actions. However, these projects can lead into a pseudo-activism for lazy people as well. Keywords: Civic participation, Cyber-democracy, Gamification narratives, Play, Civic empowerment, Activism, Gamification.
\end{abstract}

Sumario. 1. Introducción. 2. La participación ciudadana en entornos digitales: el ideal de ciberdemocracia. 3. Play level up: aproximación teórica a las narrativas gamificadas. 4. Metodología. 5. Resultados. 5.1. Resultados del análisis de "Fort McMoney". 5.2. Resultados del análisis de "A Force More Powerful". 6. Conclusiones. 7. Referencias bibliográficas.

Cómo citar: Pérez-Escola M.; Navazo Ostúa P. (2019). Activismo y narrativas gamificadas: estudio comparativo de entornos ciberdemocráticos de empoderamiento ciudadano, en CIC. Cuadernos de Información y Comunicación 24, 31-46.

$1 \quad$ Universidad Loyola Andalucía. 


\section{Introducción}

Los procesos comunicativos tradicionales, propios de la cultura de masas (Lasswell, 1948; Macdonald, 1969; Mannheim, 1964; Hegel, 1968), se caracterizaban, principalmente, por su hermetismo institucional y su opacidad informativa. Estos sistemas se articulaban siguiendo una estructura lineal por donde discurrían flujos de información unidireccionales en los que participaban tres únicos actores: el emisor, el mensaje y el receptor. Este modelo de comunicación no admitía que el receptor pudiera interactuar con el emisor o con el mensaje: los ciudadanos -receptores- eran una masa inerte que consumía contenidos politizados a través de los medios de comunicación de masas -radio, prensa y televisión-, mientras que las élites de poder -emisor- eran quienes tenían pleno control para decidir qué decir, en qué momento y a quién.

No obstante, el ciudadano posmoderno (Lipovetsky, 2006; Bauman, 2007; Castells, 2008) ya no pertenece a esa multitud homogénea propia de la modernidad. Las sociedades occidentales han evolucionado hacia una nueva forma de organización en la que el individuo muestra su disposición y su interés por involucrarse y participar activamente en la vida política (Kollock \& Smith, 1995; Leadbetter, 1999; Hall, 1999; Lim, 2002; Chadwick, 2003; Dahlgren, 2011; Freire, 2013). La revolución digital y tecnológica ha contribuido de manera decisiva a esta transformación (Coleman \& Blumler, 2009). Las relaciones entre ciudadanos y élites de poder han cambiado. El arquetipo de Lasswell (1948) y el modelo panóptico de Bentham han quedado obsoletos en este nuevo ecosistema participativo.

La convergencia digital (Jenkins, 2006) ha supuesto la redefinición de las funciones del ciudadano y ha motivado la creación un modelo panóptico invertido: los ciudadanos tienen pleno poder para moldear su participación en el escenario global colaborativo. Sin embargo, desde una perspectiva más pesimista, el panóptico invertido también "hace posible una red donde cada uno vigila al otro, todos se vigilan y el modelo termina en una "vigilancia sin guardián". Uno de los mecanismos que permite este funcionamiento es el Big Data" (Mallamaci, 2017: 83). En este sentido, si el Big Brother de George Orwell describía una sociedad vigilada por un único ente, ahora, el Big Data o Big Brother digital facilita que todos los ciudadanos monitoricen al resto de ciudadanos (Mallanaci, 2017).

Además, aunque esta convergencia digital ofrezca nuevas oportunidades y herramientas de colaboración e implicación, no todos los ciudadanos se convierten en activistas digitales o ciberactivistas: el hecho de que la digitalización traiga nuevas posibilidades de participación no implica que el sujeto esté motivado, sepa o quiera participar. No todos los usuarios desean involucrarse en la esfera pública y no todos los ciberactivistas se comprometen al mismo nivel (Sampedro et al., 2014).

En este sentido, surge la necesidad de conocer si existen narrativas de participación ciudadana que revistan un mayor atractivo para la sociedad que otras y que, de alguna forma, también estimulen a los ciudadanos a participar y comprometerse como activistas sociales. Siguiendo esta línea, el objetivo principal de este estudio busca analizar si las narrativas gamificadas pueden convertirse en una valiosa herramienta de empoderamiento ciudadano con la que motivar la participación de individuo en entornos ciberdemocráticos.

Los sistemas gamificados se articulan siguiendo la estructura lógica del juego, lo que constituye un importante reclamo para captar la atención del público: por un 
lado, la naturaleza de cualquier juego consigue conectar con el individuo desde un punto de vista emocional, físico o mental. Por otro lado, el ser humano es, por naturaleza, es un "homo ludens" (Huizinga, 1949) que siente devoción por el juego y los contenidos lúdicos.

Con este fin, se ha seleccionado un conjunto representativo de dos productos gamificados de corte activista: "Fort McMoney", que es un i-doc (Gaudenzi, 2013; Gifreu, 2011; Nash, 2011) que narra la vida de los habitantes de la ciudad canadiense de Fort McMurray -cuya economía se basa en la explotación petrolífera-, y "A Froce More Powerful", un serious game (Jenkins, 2007) que nació a raíz del libro de York Zimmerman"s Steve York, en 1997, y que supone un instrumento de aprendizaje gamificado que enseña cómo resolver movimientos políticos y sociales no violentos.

\section{La participación ciudadana en entornos digitales: el ideal de ciberdemocracia}

La participación ciudadana es un elemento esencial para el desarrollo y evolución de las sociedades democráticas. Para Carpentier (2012), la participación ciudadana implica dos conceptos esenciales: "acceso" e "interacción". El "acceso" favorece que el individuo contacte y reciba una respuesta de los actores e instituciones políticas. En este sentido, el acceso es la habilidad de la sociedad para acercarse más a los sistemas comunicativos (Berrigan, 1979: 18) y conlleva reforzar la presencia (Carpentier, 2015: 10) de la comunidad.

Por otro lado, la "interacción" implica una relación sociocomunicativa (Carpentier, 2012) entre ciudadanos que crean, comparten, seleccionan e interpretan todo tipo de contenidos e información (Jenkins y Carpentier, 2013). La interactividad es, por tanto, una característica intrínseca de los escenarios digitales, ya que facilita que el individuo interactúe con otros usuarios de la red (Castells, 2008) o, incluso, con la propia plataforma tecnológica (Carpentier, 2015).

En líneas generales, la participación ciudadana representa una forma de interacción social que implica un alto nivel de compromiso y motivación cívica. La participación ciudadana está incrustada en la realidad política y la lógica democrática de la sociedad contemporánea (Carpentier, 2012); es un componente para el buen gobierno y el instrumento con el que los individuos adquieren poder para influir en las decisiones políticas, intervenir en los asuntos públicos, cambiar dictámenes y sentencias o, incluso, para incentivar la renovación del sistema democrático.

No obstante, para que la participación ciudadana pueda desarrollarse plenamente es necesario diseñar de un sistema de organización social y político basado en el autogobierno digital y la soberanía popular, ya que los tradicionales modelos de democracia representativa se han convertido en estructuras deficientes que no satisfacen las necesidades e intereses de la sociedad contemporánea; el sistema representativo se caracteriza por lo que Bobbio (1986) denomina como las "falsas promesas" y "obstáculos" de la democracia. El individuo no solo se muestra escéptico y desconfía de la actuación de los actores políticos (Presno Linera, 2012), sino que ha desarrollado un sentimiento de indignación colectiva que alimenta el deseo de instaurar una nueva lógica y valores políticos.

En este contexto, surge la necesidad de instaurar un modelo ciberdemocrático (Bellamy, 2000) como construcción alternativa que resarza las debilidades que pre- 
senta la democracia representativa y que, a la vez, otorgue más oportunidades de participación directa ciudadana (Hamilton, Madison \& Jay, 2012). La ciberdemocracia nace de la evolución de la democracia deliberativa (Martí, 2008; Ganuza, 2012) y participativa (Dahlgren, 2013; Lévy, 2004) e implica un cambio estructural democrático a través de las nuevas tecnologías de la comunicación: la asimetría de poderes que existe entre ciudadanos y gobierno empieza a diluirse en este ecosistema socio-político.

Este modelo digital no solo supone reforzar el poder ciudadano sobre las tradicionales instituciones políticas, sino que además se ha convertido en el arquetipo representativo de la sociedad red (Castells, 2008). Sin embargo, el valor de la ciberdemocracia y de la sociedad red no solo radica en la multiplicidad de redes, sino en la utilidad que los ciudadanos les dan a esas conexiones; es decir, las comunidades virtuales deben alimentar o "polinizar" esas redes con palabras, diálogo y acciones en común (Rincón, 2015). De lo contrario, las redes caerán en desuso y morirán y, con ello, también la ciberdemocracia.

En definitiva, la ciberdemocracia es un modelo de empoderamiento ciudadano que se articula como una adhocracia (Toffler, 1973) digital, donde los individuos monitorizan las actuaciones del gobierno (Schudson, 1998; Keane, 2009; Feenstra \& Casero-Ripollés, 2014) y muestran su interés por implicarse en el proceso de toma de decisiones políticas y sociales.

Sin embargo, desde una perspectiva crítica, Morozov (2009) advierte que este tipo de participación política ciudadana puede desembocar en slacktivism o activismo para vagos; es decir, el individuo se siente orgulloso y feliz cuando descubre que puede hacer algo bueno por la sociedad sin que ello requiera implicarse en política, ni participar en alguna protesta, ni recaudar o invertir dinero (Neumayer \& Schoßböck, 2011). Morozov (2009) denomina a este sentimiento el factor feel-good y es la razón principal que explica la popularidad del slacktivism, así como el catalizador de una sociedad indolente o "lazy generation" (Morozov, 2009) que quiere participar y cambiar el mundo sin esfuerzo alguno.

Además, siguiendo este planteamiento crítico, a pesar de que la ciberdemocracia ofrezca nuevas oportunidades y herramientas de colaboración e implicación, no todos los ciudadanos desean participar en la esfera pública (Dalton, Bürkin \& Drummond, 2001). En este contexto, se considera que las narrativas gamificadas pueden representar un valioso catalizador del activismo, ya que favorecen la implicación y participación del ciudadano a través del juego. El juego forma parte de la idiosincrasia del individuo y es un elemento inherente a la cultura humana (Huizinga, 1949): lo lúdico ya no solo se presenta como un fenómeno reservado para niños, sino que se ha convertido en una actitud social cotidiana: "la marca del adulto posmoderno es la inclinación de acercarse al juego con entusiasmo, como hacen los niños" (Bauman, 1995: 99).

\section{Play level up: aproximación teórica a las narrativas gamificadas}

La gamificación en una valiosa herramienta que está introduciéndose en diferentes sectores sociales, económicos y políticos con la intención de captar la atención, mo-

The mark of postmodern adulthood is the willingness to embrace the game whole-heartedly, as children do. 
tivar y comprometer a los usuarios de cualquier audiencia contemporánea. El principal motivo por los que agregar elementos propios de la gamificación dentro de una estrategia general de comunicación es que la gamificación se fundamenta en las emociones humanas, así como en la tendencia natural de las personas a la socialización, el aprendizaje, la competición y el progreso.

Sin embargo, a pesar de que la gamificiación está presente en múltiples escenarios contemporáneos, este fenómeno resulta ser un concepto polisémico que denota multitud de significados e interpretaciones. Para Werbach \& Hunter (2012: 25), el término «gamificación» apareció, por primera vez, en el año 2003, aunque no fue hasta 2010 cuando esta locución comenzó a adquirir popularidad entre los investigadores (Werbach \& Hunter, 2012; Deterding, et al., 2011). En su acepción más simple, la gamificación ha consistido en introducir elementos divertidos y competitivos, propios del mundo de los juegos y videojuegos, en las narrativas actuales propias del marketing, la educación o la participación ciudadana. En un principio, las prácticas de la gamificación se centraban en alentar a sus usuarios a realizar una serie de tareas para generar conversaciones monetarias, aumentar la base de participantes activos o suscitar lealtad a unos determinados productos o servicios.

En cambio, la gamificación, en la actualidad, busca fomentar experiencias memorables de participación directa, así como en crear una práctica que suponga un desafío pedagógico e interactivo para el usuario. Esto implica que, hoy en día, la gamificación va más allá de incorporar elementos propios de la diversión o la competición a un contenido narrativo, pues, actualmente, existen numerosas maneras en que la gamificación puede ser implementada en la sociedad. Zichermann \& Cunningham (2011: 14) definen la gamificación como un proceso que se desarrolla, sobre todo, en contextos no lúdicos y donde se emplean "serious games, advergaming y juegos para el cambio"2. Por tanto, la gamificación utiliza la lógica y las herramientas propias del juego -game thinking y game mechanics- para comprometer al usuario y solucionar problemas (Zichermann \& Cunningham, 2011).

De forma similar, Werbach \& Hunter (2012: 26) coinciden con Zichermann \& Cunningham (2011) en que la gamificación tiene que ver con el uso de técnicas, elementos y diseños propios del juego aplicados en contextos no lúdicos. En esta línea, Kapp (2012: 10) ofrece una definición más amplia y explica que la gamificación implica "utilizan mecánicas, estéticas y lógicas basadas en el juego para implicar a la gente, motivar la acción, promover el aprendizaje y resolver problemas "3. Así pues, la gamificación se resume como la integración de dinámicas y elementos propios del juego en escenarios que no guardan relación con el juego -como es el activismo, que es el caso que nos ocupa en esta investigación-.

Existe, en este punto, una falta de consenso entre los investigadores (Juul, 2003; Wilson et. al., 2009; Kapp et al., 2013; Kiryakova et al., 2014) sobre cuáles son los elementos o características propias del juego que resultan imprescindibles en las narrativas gamificadas. En cualquier caso, la cuestión central no radica en saber qué elementos lúdicos conforman los sistemas gamificados, sino en saber combinar estos elementos y dinámicas para alcanzar un mayor nivel de motivación e implicación del usuario.

Serious games, advergaming, and games-for-change.

Using game-based mechanics, aesthetics and game thinking to engage people, motivate action, promote learning, and solve problems 


\section{Metodología}

La propuesta metodológica que presenta este estudio se centra en realizar un análisis comparativo de dos productos gamificados: "Fort McMoney" y "A Force More Powerful". Estos dos casos de estudio se consideran representativos del fenómeno de la gamificación y constituyen, sobre todo, una significativa herramienta de empoderamiento ciudadano: "Fort McMoney" es un i-doc (Gaudenzi, 2013; Gifreu, 2012; Nash, 2011) ludificado que incentiva la participación del jugador en la esfera política local. En cambio, "A Force More Powerful" es un serious game que enseña a los activistas a adquirir, mediante ejercicios de entrenamiento y de organización, las habilidades necesarias para impulsar un cambio social o movimiento político no violento. En definitiva, como se puede comprobar, a pesar de que la naturaleza de los productos es distinta, ambas narrativas se enmarcan en el ámbito de la participación política ciudadana.

Para analizar estos productos, se ha recurrido a una metodología cualitativa que hibrida la técnica Octalysis (Chou, 2015) con el modelo de la Escalera de participación (Arnstein, 1969). El objetivo de este maridaje es doble: por un lado, a través del método Octalysis, se busca identificar y definir la principal motivación que mueve a los usuarios a participar en estas narrativas gamificadas. Para ello, se ha estudiado el diseño de los dos productos gamificados seleccionados con el fin de conocer los factores que activan el engagement del usuario (O'Brien \& Toms, 2008). En este sentido, Chou (2015) identifica ocho ejes impulsores o "core drivers" que están presentes, de una forma más o menos notable, en los productos gamificados:

Figura 1. Modelo Octalysis de Chou (2015).

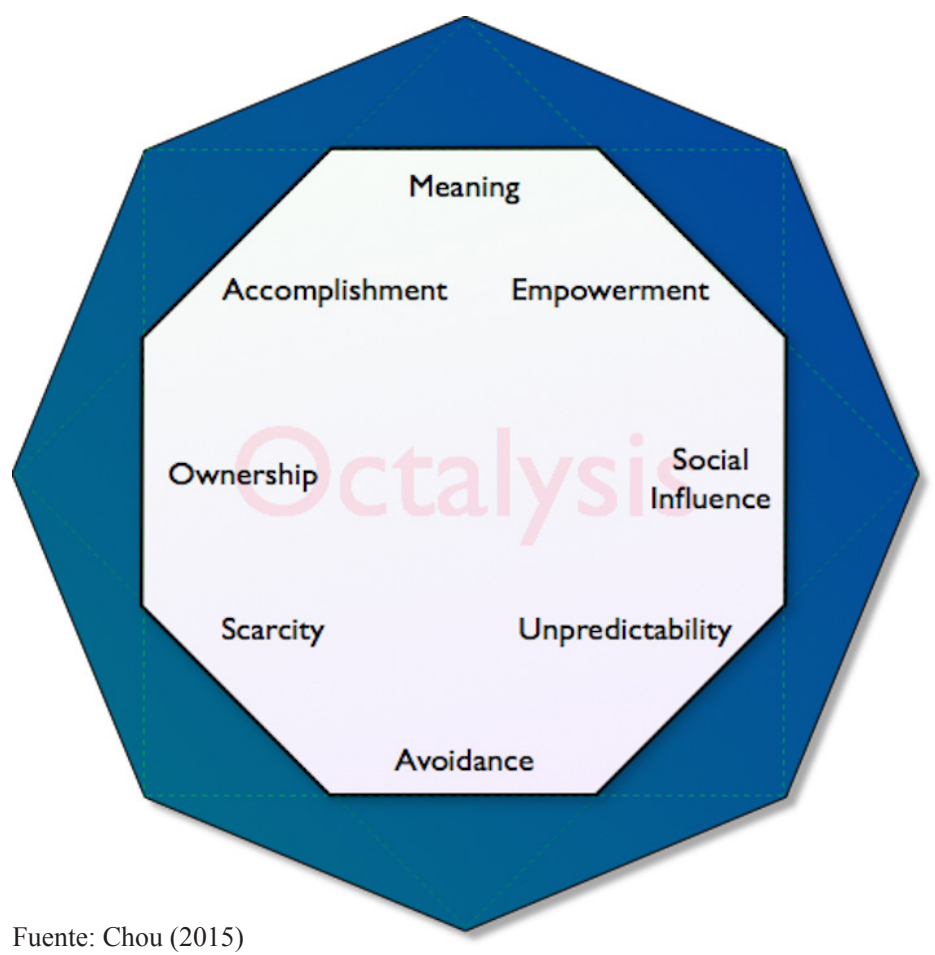


- Epic meaning \& calling - Significado épico y llamada: esta estrategia impulsora se basa en transmitir a los usuarios la sensación de que están implicados en algo de gran trascendencia; la narrativa gamificada invita al usuario a pensar que han sido elegidos para llevar a cabo una importante misión y que sus acciones tienen una relevancia inconmensurable como ocurre, por ejemplo, en Wikipedia.

- Development \& accomplishment - Desarrollo y cumplimiento: esta motivación se refiere a la predisposición natural del ser humano a progresar, desarrollar habilidades y, finalmente, superar nuevos retos o desafíos. Este core driver se consigue a través de elementos más tangibles como insignias, puntos o leaderboards que, de un modo u otro, recompensan la experiencia adquirida por el usuario y potencian su participación en la narrativa.

- Empowerment of creativity \& feedback - Empoderamiento de la creatividad y retorno: esta motivación está vinculada a la satisfacción que los usuarios experimentan cuando pueden participar e involucrarse en el proceso creativo de la narrativa: crear nuevas realidades, diseñar ecosistemas o, incluso, transformar el entorno, como así ocurre, por ejemplo, en Lego.

- Ownership \& possession - Propiedad y posesión: este eje impulsor se basa en transmitirles a los usuarios la sensación de que poseen algo valioso. En la mayoría de los casos, cuando esto ocurre, el individuo empieza a invertir tiempo en personalizar ese producto, en mejorarlo. De esta forma, cuanto más tiempo dedica al producto, más motivado se siente y desarrollo un elevado sentimiento de posesión y propiedad hacia el producto como ocurre, por ejemplo, con el Tamagotchi.

- Social influence \& relatedness - Influencia social y relación: este eje impulsor agrupa todos los elementos sociales que motivan a los individuos: la tutoría, la aceptación, el compañerismo, la respuesta social y la envidia. Este core driver se basa en la premisa de que el ser humano es, por naturaleza, un ser social que disfruta cuando está en familia o con sus amigos.

- Scarcity \& impatience - Escasez e impaciencia: este eje motivador se basa en el estímulo de querer conseguir algo por la simple razón de que es difícil de obtener o porque todavía no se ha conseguido.

- Unpredictability \& curiosity - Impredecibilidad y curiosidad: este core driver asume que la sorpresa es un elemento que origina motivación: cuando los usuarios no saben qué va a pasar, se comprometen, de forma inconsciente, con la narrativa gamificada para conocer qué ocurre al final. Este eje impulsor es el principal aliciente de muchas películas y libros.

- Loss \& avoidance - Pérdida y evitación: este eje impulsor se basa en la motivación que deriva del miedo a perder algo o a que ocurran hechos indeseados. El usuario de Farmville, por ejemplo, se implica en juego porque si no atiende y cuida sus cosechas, corre el riesgo de perder sus bienes y ganancias.

Por otro lado, el segundo objetivo de esta investigación es conocer el grado de participación que ofrecen las narrativas de los dos casos de estudio seleccionados -"Fort McMoney" y “A Force More Powerful"-. Para ello, se ha recurrido al paradigma de la Escalera de la participación de Arnstein (1969) que refleja, de un modo metafórico y crítico, los diferentes niveles de participación ciudadana que pueden prosperar en cualquier sistema social. 
Figura 2. Escalera de la participación de Arnstein (1969).

\begin{tabular}{|l|l} 
8. Control ciudadano & \multirow{2}{*}{ CONTROL CIUDADANO } \\
\hline 7. Delegación del poder & \\
\hline 6. Colaboración & \\
\hline 5. Asesoría & CORTINA DE HUMO \\
\hline 4. Consulta & \\
\hline 3. Información & \\
\hline 2. Terapia & NO PARTICIPACIÓN \\
\hline 1. Manipulación &
\end{tabular}

Fuente: Arnstein (1969)

Los dos primeros estribos de esta escalera constituyen la base del paradigma y representan las formas de "no participación". Según Arnstein (1969), estos escalones simbolizan una comunidad de usuarios manipulados que no pueden acceder a los espacios de participación. Las élites de poder asumen el papel de educadores o "terapeutas" de los ciudadanos para construir una sociedad controlada y supeditada a la influencia del creador del producto. En cierto sentido, este ecosistema social que se crea -en el que los ciudadanos viven una realidad distorsionada- es parecido al régimen totalitario que George Orwells describe en su novela 1984.

Los tres siguientes peldaños corresponden a la "información", la "consulta" y la "asesoría". En este espacio, al que Arnstein (1969) denomina "tokenism" o "cortina de humo", se desarrolla una participación alegórica; es decir, los usuarios tienen la oportunidad de expresar sus opiniones y propuestas, pero no disfrutan del poder necesario para tomar sus propias decisiones. Es una estrategia de distracción, en la que se despliegan canales de comunicación unidireccionales, que impide que la comunidad social pueda interactuar con la narrativa gamificada.

Finalmente, la cúspide de esta escalera está conformada por tres niveles que describen las distintas formas de "control ciudadano". En estos últimos escalones, el individuo adquiere poder para participar activamente en la narrativa gamificada, de forma que puede crear personalizar su implicación: puede crear nuevos espacios, 
modificar los ya existentes, personalizar elementos, etc. La participación ciudadana se entiende, por tanto, como un instrumento que otorga poder al individuo (Carpentier, 2012) y permite que los usuarios defiendan conjuntamente los intereses y necesidades colectivas. Se crea así una suerte de colaboratorio donde los usuarios moldean su participación para personalizar su experiencia en la narrativa de los productos gamificados. De esta forma, la narrativa gamificada se convierte en un commodity que otorga plena libertad al usuario para participar y actuar.

\section{Resultados}

En este apartado se presentan los principales resultados obtenidos tras el análisis de "Fort McMoney" y "A Force More Powerful" en relación con la técnica Octalysis (Chou, 2015) y el modelo de la Escalera de la participación (Arnstein, 1969). Según los datos obtenidos, "Frot McMoney" está más gamificado, es más ciberdemocrático $y$, por tanto, ofrece un mayor nivel de participación que "A Force More Powerful", a pesar de que ambas narrativas se consideran importantes herramientas de empoderamiento ciudadano.

\subsection{Resultados del análisis de "Fort McMoney"}

"Fort McMoney" es un documental interactivo (Gaudenzi, 2013; Gifreu, 2011; Nash, 2011), dirigido por David Dufresne, que muestra y narra los problemas diarios a los que se enfrentan los ciudadanos de Fort McMurray, una ciudad canadiense que basa su economía en la explotación petrolífera. El popular video-documental Fort McMoney se presentó en el Festival Internacional de Cine de Ámsterdam en 2013, con eventos previos al lanzamiento en los festivales documentales de París, Toronto y Montreal. Aclamado por el New York Times como "el matrimonio perfecto entre el cine y el videojuego", su estreno contó con el respaldo de la crítica internacional por su originalidad y su cuidada producción. Entre estos reconocimientos, Fort McMoney fue premiado, en los Premios Nacionales de Canadá en febrero de 2015, como la mejor producción interactiva para medios digitales.

Este documentary game, que posee una interfaz que simula un videojuego, permite al usuario interactuar con los diferentes elementos que componen la experiencia. De esta forma, a la misma vez que el jugador se involucra y progresa en la historia -participa en la construcción del relato-, también puede chatear con otras personas que estén en línea en ese momento, buscar pistas, entrevistar a los habitantes del juego, descubrir nuevos lugares, interactuar con su panel de control para ver qué misiones tiene pendientes, proponer debates y referéndums para decidir cómo evolucionará el juego durante las próximas semanas, etc. Así, cuanto más juegue el usuario y, por tanto, cuanto más conozca sobre la realidad de Fort McMurray, más poder tendrá el jugador y más influyentes serán sus comentarios entre la comunidad de jugadores.

Ahora bien, siguiendo los ocho ejes impulsores o "core drivers" que propone Chou (2015) en su técnica Octalysis, se comprueba que, en "Fort McMoney", no existe ningún tipo de elemento característico de los motores motivacionales de la propiedad -ownership \& possession- ni de la escasez -scarcity \& impatience-. Existe una escasa presencia del miedo a la pérdida-loss \& avoidance-de las áreas 
naturales protegidas y del temor a perder los grandes beneficios que genera el tejido industrial. Por otro lado, el producto tiene un alto potencial empoderador-empowerment of creativity \& feedback-porque permite al usuario utilizar su capacidad creativa para elegir la trama narrativa.

El proyecto ofrece distintas posibilidades para resolver el argumento y crea un suspense narrativo que suscita gran curiosidad en el usuario por conocer los posibles acontecimientos del relato. Además, el principio de sentido o significación - epic meaning \& calling- está agudamente presente, pues, a través del activismo y el compromiso con una causa ideológica, la acción narrativa está en sintonía con los valores personales del usuario. Finalmente, resulta igualmente importante el principio de realización -development \& accomplishment-, tanto en la exploración de una realidad remota, como en la consecución de los logros insertos en la trama narrativa.

Figura 3. Aplicación del Octalysis (Chou, 2015) en el proyecto "Fort McMoney".

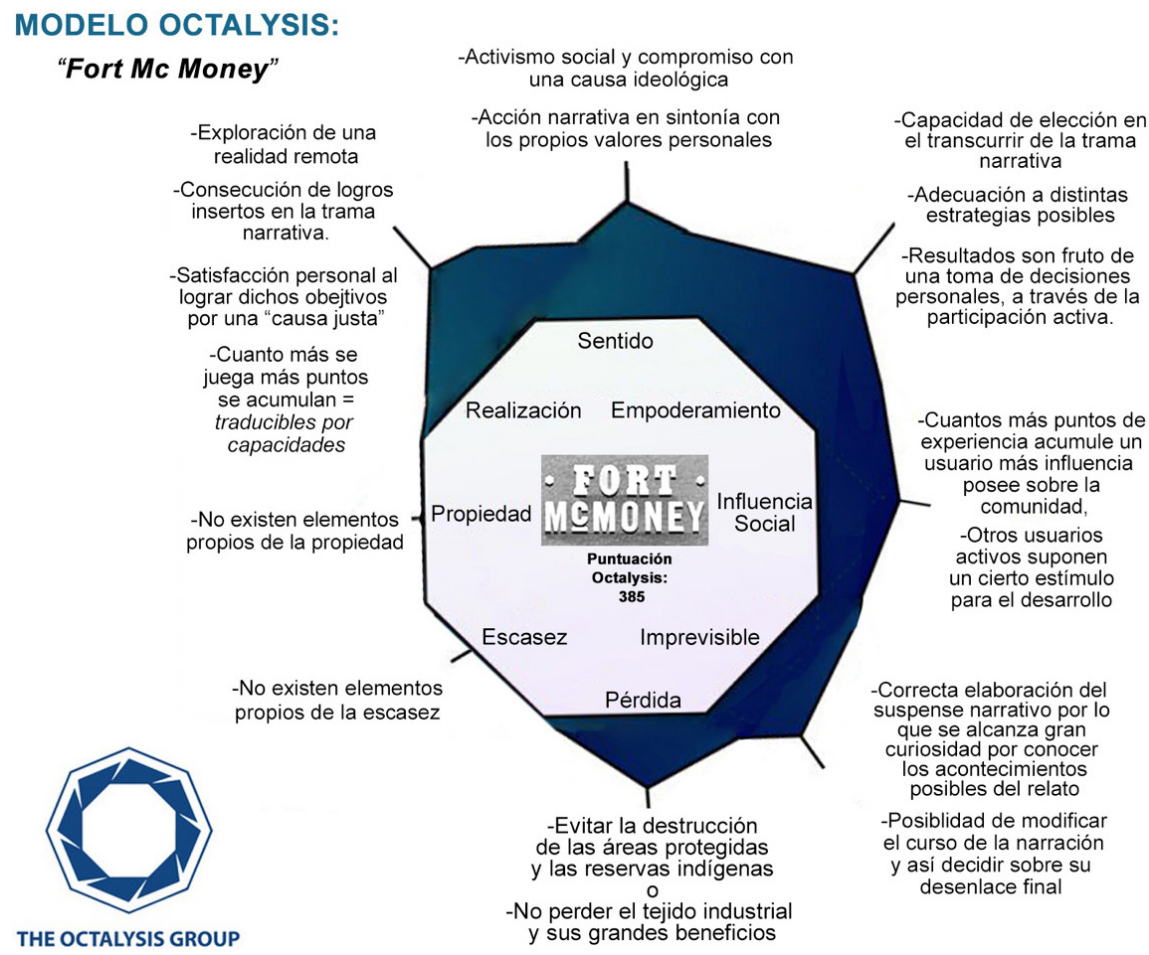

Fuente: Elaboración propia a partir del modelo Octalysis de Chou (2015).

Desde la perspectiva del modelo de la Escalera de la participación (Arnstein, 1969), el proyecto "Fort McMoney" se sitúa en séptimo peldaño del último bloque de la escalera: "delegación de poder". En este caso, el usuario tiene un alto grado de control sobre el producto porque cada jugador puede tomar decisiones que modifican su experiencia, puede enviar iniciativas al Ayuntamiento para crear un cambio social, puede intercambiar opiniones con otros jugadores, etc. Sin embargo, el usuario no tiene plena autoridad para cambiar por completo la narrativa: el jugador puede adaptar su experiencia con el producto, pero no puede cambiar por completo el entorno preestablecido. 
Por tanto, existe una delegación de poder, pero no un control total: la narrativa gamificada de "Fort McMoney" presenta múltiples opciones para cambiar el relato, pero la experiencia del usuario es el resultado de un proceso meditado previamente $\mathrm{y}$, de alguna forma, consentido por el juego. El usuario no puede proponer o incluir elementos que no hayan sido anticipados por el propio juego. Por tanto, a pesar de que el producto ofrece una falsa sensación de pleno control -el jugador puede cambiar la estructura y contenido del documental-, la realidad es que el usuario sigue teniendo una autonomía limitada. Aun así, "Fort McMoney" representa un escenario ciberdemocrático que corresponde a una forma de "open model" (Cortés-Selva \& Pérez-Escolar, 2016) o "living documentary" (Gaudenzi, 2013), ya que acepta las contribuciones individuales de los jugadores y ofrece un significativo nivel de autogestión al ciudadano. En este sentido, "Fort McMoney" se convierte en una especie de adhocracia (Toffler, 1973) digital que favorece la interacción y el debate entre los agentes sociales y el gobierno.

\subsection{Resultados del análisis de "A Force More Powerful"}

"A Force More Powerful" es un serious game que surgió a partir del libro que York Zimmerman's Steve York escribió, en 1997, bajo el mismo nombre. “A Force More Powerful" se desarrolló en asociación con el Centro Internacional de Conflictos No Violentos, cuyo objetivo era desarrollar un juego que capacitaría a los activistas con las habilidades de planificación y ensayo necesarias para organizar un movimiento de resistencia no violento para el cambio político y social. Podría decirse que ya, desde su lanzamiento, en la Feria Internacional de Serious Games Summit en Washington D.C., "A Force More Powerful" fue uno de los productos más esperados y aclamados de la feria, ha sido galardonado con numerosos premios y se ha traducido a diez idiomas y distribuido en todo el mundo.

Este videojuego interactivo simula escenarios y sucesos de la vida real para enseñar a los usuarios a resolver conflictos y enfrentamientos de forma no violenta. Estas simulaciones pretenden ser consistentes con la realidad, empleando las herramientas técnicas de las dinámicas para apoyar dicha exploración en determinados micromundos o como una herramienta de construcción de los mismos (Papert, 1980). Tal y como ya apuntaría Baudrillard, una simulación se presenta como una abstracción interactiva, o como una simplificación, de algo de la vida real (Baudrillard, 1983).

En este sentido, "A Force More Powerful" es instrumento de aprendizaje gamificado que ayuda a los jugadores a adquirir las habilidades necesarias para desarrollar estrategias y tácticas que generen movimientos políticos no violentos. Este tipo de activismo es una forma de desobediencia civil (Bedau, 1961; Habermas, 2002; Magaloni, 1990; Marcone, 2009; Rawls, 1979; Thoureu, 2002) muy común en las sociedades occidentales para desafiar y combatir las injusticias sociales.

En líneas generales, "A Force More Powerful" permite a los jugadores tomar decisiones, elaborar estrategias, reclutar personajes, crear alianzas, etc. Todo ello para enseñar al usuario a combatir la opresión con métodos no violentos. El juego presenta, además, diez escenarios diferentes -inspirados en contextos históricosque influyen en las tácticas y decisiones que tome el usuario.

Ahora bien, siguiendo los ocho ejes impulsores o "core drivers" que propone Chou (2015) en su técnica Octalysis, se comprueba que, en "A Force More Power- 
ful" genera un alto grado de satisfacción simbólica-development \& accomplishment-, ya que los usuarios tienen que ir cumpliendo los objetivos propuestos en cada escenario sociopolítico. Del mismo modo, el diseño del juego también favorece la sensación de progreso, crecimiento y mejora de las habilidades del usuario a lo largo de la narrativa.

Figura 4. Aplicación del Octalysis (Chou, 2015) en el proyecto "A Force More Powerful”.

MODELO OCTALYSIS:

\section{"A FORCE MORE POWERFUL"}

-Satisfacción simbólica en la consecución de los objetivos sociales marcados para cada escenario.

-Correcto desarrollo en la sensación de progreso crecimiento y mejora de las habilidades del usuario con el avance del juego.

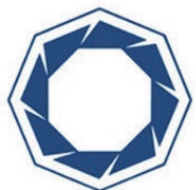

-Nula sensación de propiedad sobre ningún aspecto de la dinámica de juego.

to

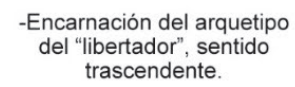

-Puesta a prueba de habilidades en un marco de trascendencia.

aro,
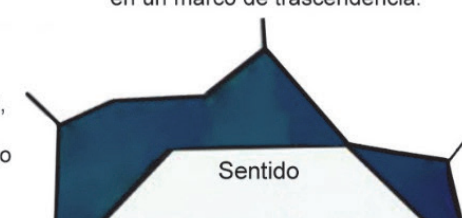
sea superar y conocer los subsiguientes escenarios y misiones. Sin embargo, en la narrativa de este juego no se encuentra ningún eje impulsor basado en la influencia social -social influence \& relatedness-. Aunque el core drive de la creatividad-empowerment of creativity \& feedback-está moderadamente presente en la dinámica del juego, ya que el usuario necesita concebir y crear diversas estrategias para resolver todo tipo de conflictos. Finalmente, el eje impulsor del significado épico y llamada -epic meaning \& calling- está moderadamente presente en este serious game. Tal y como está planteada la dinámica de juego, el usuario experimenta un proceso de transfiguración desde un ente pasivo a un sujeto activista, líder de un movimiento de protesta en una situación de injusticia social. Por tanto, este cambio fomenta que el usuario asimile el rol de sujeto libertador. De igual modo, la propia dinámica y desarrollo de juego, que se basa probar las habilidades estratégicas del usuario en un determinado marco sociopolítico simulado, aviva el interés, el aprendizaje y la sensibilización del usuario sobre el propio ámbito del activismo político y la lucha social.

Desde la perspectiva del modelo de la Escalera de la participación (Arnstein, 1969), el proyecto "A Force More Powerful" se sitúa en un estadio intermedio, en concreto, en el peldaño "consulta" del bloque "cortina de humo". En este caso, el proyecto infunde, en el usuario, la sensación de que su experiencia es única como consecuencia de las decisiones que toma a lo largo del juego, pero, en realidad, la narrativa es prácticamente idéntica para cada usuario. Por ello, este serious game representa un escenario ciberdemocrático que se identifica como un "semi-closed model" (Cortés-Selva \& Pérez-Escolar, 2016), ya que la estructura del producto es hermética y limitada, pero permite al jugador investigar diferentes posibilidades e indagar en el desarrollo del relato. El individuo puede planificar su propia ruta, estrategias y aliados, pero no puede modificar ni crear nuevos entornos o la estructura del producto, es decir, que el jugador solo puede cambiar ciertos aspectos de la historia, pero siempre y cuando la narrativa del juego lo permita.

Así pues, "A Force More Powerful”" se presenta como una plataforma interactiva que consulta o pregunta al usuario la opción que quiere elegir de entre las que el propio juego le ofrece de forma predeterminada. El jugador queda así imbuido en una atmósfera pesudoparticipativa -cortina de humo-, porque el usuario puede personalizar su escenario de juego y decidir cómo encaminar la narrativa, pero su participación es limitada porque las decisiones que tome han sido previamente establecidas y determinadas por la plataforma. Así pues, a pesar de que el individuo puede personalizar ciertos aspectos en el juego -como las tácticas, las estrategias o el escenario de juego-, la narrativa de este producto -inicio, nudo y desenlace- se desarrolla de la misma forma en cada experiencia.

\section{Conclusiones}

Los resultados obtenidos en este estudio permiten concluir que las narrativas gamificadas, como "Fort McMoney" y "A Force More Powerful", constituyen un renovado aliciente para la participación ciudadana que reaniman y alimentan, además, las acciones activistas. Las narrativas de los productos gamificados resultan atractivas y suscitan fascinación, ya que difieren de los tradicionales formatos de implicación social y tienen, como principal eje motivador, el juego. 
Proyectos como "Fort McMoney" y "A Force More Powerful" representan herramientas de empoderamiento ciudadano porque motivan el aprendizaje de los usuarios durante el juego, permiten que los ciudadanos interactúen en red con otros usuarios y facilitan instrumentos reales con los que el individuo pueda emprender una campaña activista en entornos offline $u$ online. Todos estos factores originan que, en líneas generales, el usuario se sienta bien consigo mismo porque se siente realizado a través del juego: siente que el tiempo que invierte en el juego no es tiempo de ocio -de autocomplaciencia o egoísmo-, sino que es tiempo que invierte en ayudar a los demás, en aprender, en comprometerse con ciertos problemas sociales y, en definitiva, en promover un cambio social. Es lo que Morozov (2009) el factor feel-good y el principal catalizador del slacktivismo o activismo para vagos. En este sentido, las narrativas gamificadas son responsables de alentar a una generación indolente o "lazy generation" (Morozov, 2009) que quiere participar y cambiar el mundo sin esfuerzo alguno. Este pseudoactivismo conlleva ciertos riesgos porque supone aceptar que no todos los usuarios están preparados para aprovechar las oportunidades y sortear las amenazas que alberga el ciberactivismo.

\section{Referencias bibliográficas}

Arnstein, S. (1969). A ladder of citizen participation. Journal of the American Institute of Planners, 35(4), 216-224

Baudrillard, J. (1983). Simulations. Philosophy Hall Columbia University New York City, New York, U.S.A.

Bauman, Z. (1995). Life in Fragments. Essays in Postmodern Morality. Oxford: Blackwell

Bauman, Z. (2007). Miedo líquido. La sociedad contemporánea y sus temores. Barcelona: Paidós

Bellamy, C. (2000). "Modelling electronic democracy: towards democratic discourses for an information age". En J. Hoff, I. Horrocks \& P. Tops (Eds.). Democratic Governance and New Technology (pp.33-53). Londres: Routledge

Berrigan, F. J. (1979). Community communications. The role of community media in development. Paris: Unesco.

Bobbio, N. (1986). El futuro de la democracia. México: Fondo de Cultura Económica

Carpentier, N. (2012). The concept of participation. If they have access and interact, do they really participate? Fronteiras, 14(2), pp. 164-177.

Carpentier, N. (2015). Differentiating between access, interaction and participation. Conjunctions, 2(2), pp.7-28.

Castells, M. (2008). La era de la información: Economía, sociedad y cultura. Volumen I: La sociedad red. Madrid: Alianza Editorial

Chadwick, A. (2003). Bringing e-democracy back in: Why it matters for future research on e-governance. Social Science Computer Review, 21(4), 443-455

Chou, Y. K. (2015). Octalysis: the complete gamification framework. Yu-Kai Chou: Gamification \& Behavioral Design. Recuperado de https://bit.ly/2K6fZI2

Coleman, S. \& Blumler, J. (2009). The Internet and Democratic Citizenship: Theory, Practice and Policy. Cambridge: Cambridge University Press

Dahlgren, P. (2011). Jóvenes y participación política. Los medios en la Red y la cultura cívica. Telos, (89), 12-22

Dahlgren, P. (2013). From public to civic intellectuals via online cultures. Participations. Journal of Audience \& Reception Studies, 10(1), 400-404 
Deterding, S., Dixon, D., Khaled, R. \& Nacke, L. (2011). From Game Design Elements to Gamefulness: "Defining “Gamification"”. En A. Lugmayr, H. Franssila, C. Safran, \& I. Hammouda (Eds.), Proceedings of the 15th International Academic MindTrek Conference: Envisioning Future Media Environments (pp. 9-15). Nueva York: ACM.

Feenstra, R. A. \& Casero-Ripollés, A. (2014). Democracy in the Digital Communication Environment: A Typology Proposal of Political Monitoring Processes. International Journal of Communication, (8), 2448-2468

Freire, J. (2013). Gobierno Abierto. Un proyecto en construcción. Telos, (94), 44-47

Ganuza, E. (2012). "The Deliberative Challenge". En I. Ramos Vielba \& E. Campos Domínguez (Eds.), Citizenship in 3D: Digital Deliberative Democracy (pp.19-50). Madrid: Fundación Ideas

Gaudenzi, S. (2013). The living documentary: From representing reality to co-creating reality in digital interactive documentary [Doctoral thesis]. University of London.

Gifreu, A. (2012). "Nuevo modelo de no ficción interactiva móvil. Caracterización del reportaje y el documental interactivo". En I. García-Medina \& R. S. Contreras-Espinosa (Eds.). M-todos. Tendencias y oportunidades de la movilidad digital (pp. 59-66). Bercelona: Universitat de Vic

Hall, M. (1999). Virtual colonization. Journal of Material Culture, 4(1), 39-55

Hamilton, A., Madison, J. \& Jay, J. (2012). El federalista. México: Fondo de Cultura Económica Hegel, G. W. F. (1968). Filosofía del derecho. Buenos Aires: Editorial Claridad

Huizinga, J. (1949). Homo ludens. A study of the play-element in culture. London: Routledge \& Kegan Pau

Jenkins, H. (2006). Convergence Culture: Where Old and New Media Collide. Nueva York: New York University Press

Jenkins, H. (2007). From Serious Games to Serious Gaming (Part One): Revolution. Recuperado de https://bit.ly/2Vgw4RI

Jenkins, H. y Carpentier, N. (2013). Theorizing participatory intensities: A conversation about participation and politics. Convergence: The international journal of research into new media technologies, 19(3), pp. 265-286

Juul, J. (2003). "The Game, the player, the world: Looking for a heart of gameness". En M. Copier \& J. Raessens, (Eds.), Proceedings at the Level Up: Digital Games Research Conference, (pp. 30-45). Utrechet, the Netherlands: Utercht University.

Kapp, K. M. (2012). The Gamification of Learning and Instruction: Case-Based Methods and Strategies for Training and Education. Nueva York: Pfieffer: An Imprint of John Wiley \& Sons.

Kapp, K. M., Blair, L. \& Mesch, R. (2013) The Gamification of Learning and Instruction Fieldbook: Theory into Practice. Nueva York: John Wiley \& Sons.

Keane, J. (2009). The life and Death of Democracy. Londres: Simon y Schuster

Kiryakova, G., Nadezhda, A. \& Yordanova, L. (2014). "Gamification in Education”. Proceedings of 9th International Balkan Education and Science Conference. Recuperado de https://bit.ly/2y9N9mO

Kollock, P. \& Smith, M. (1995). The sociology of cyberspace: Social Interaction and order in computer communities. Thousand Oaks: Pine Forge Press

Lasswell, H. D. (1948). "The structure and function of communications in society". En L. Bryson (Ed.), The communication of ideas (pp.37-51). New York: Harper \& Row

Leadbetter, C. (1999). Living on thin air: The new economy. London: Viking

Lévy, P. (2004). Inteligencia Colectiva. Por una antropología del ciberespacio. Washington: Organización Panamericana de la Salud 
Lim, M. (2002). Cyber-civic space in Indonesia: From panopticon to pandemonium? International Development Planning Review, 24(4), 383-400

Lipovetsky, G. (2006). Los tiempos hipermodernos. Barcelona: Editorial Anagrama

Macdonald, D. (1969). "Masscult y midcult”. En D. Macdonald, E. Shils, C. Greenberg, L. Lownthal, P. F. Lazarsfeld \& R. K. Merton (Eds.), La industria de la cultura (pp.67-156).) Madrid: Alberto Corazón

Mallamaci, M. G. (2017). El poder psicopolítico en las sociedades postdisciplinarias del homo digitalis. Revista Latina de Sociología (RELASO), 7(1), 74-94

Apuntes sobre el pensamiento de Byung-Chul Han

Mannheim, K. (1964). Ensayos de sociología de la cultura. Madrid: Aguilar

Martí, J. L. (2008). Alguna precisión sobre las nuevas tecnologías y la democracia deliberativa y participativa. Revista de Internet, Derecho y Politica, (6), 3-12

Morozov, E. (2009, 19 de marzo). The Brave New World of Slacktivism. Foreign Policy. Recuperado de http://goo.gl/EbRPNb

Nash, K. (2011). Clicking on the real: telling stories and engaging audiences through interactive documentaries. Recuperado de http://goo.gl/Vht9oc

Neumayer, C. \& Schoßböck, J. (2011). "Political lurkers? Young people in Austria and their political life worlds online”. En P. Parycek, M. J. Kripp \& N. Edelmann (presidencia). CeDEM 2011. Conferencia llevada a cabo en E-Democracy and Open Government (pp. 131-143), Danube University Krems, Austria.

O’Brien, H. L. \& Toms, E. G. (2008). "What is user engagement? A conceptual framework for defining user engagement with technology". Journal of the American society for Information Science and Technology, 59(6), 938-955

Papert, S. (1980). Tormentas de Mentes (Mindstorms), Niños, Computadoras e Ideas poderosas. Cambridge, Massachusetts. U.S.A.

Presno Linera, M. A. (2012). ¿Nos representan o no? Anales de la Cátedra Francisco Suárez, (46), 93-109

Rincón, O. (2015, mayo). PoliTICs: Del polinizar al politizar. Travesía, (33), 11-16

Sampedro, V., Sánchez, J. M. \& Campos, E. (2014). "Participación ciudadana en las cibercampañas electorales. Debates teóricos y una aproximación tipológica". En R. Cotarelo \& J. A. Olmeda (Eds.), La democracia del siglo XXI. Política, medios de comunicación, internet y redes sociales (pp. 51-80). Madrid: Centro de Estudios Políticos y Constitucionales.

Schudson, M. (1998). The Good Citizen: A History of American Public Life. Nueva York: The Free Press

Toffler, A. (1973). El shock del futuro. Barcelona: Plaza \& Janes

Werbach, K. \& Hunter, D. (2012). For the Win: How Game Thinking Can Revolutionize Your Business. Philadelphia: Wharton Digital Press.

Wilson, K. A., Bedwell, W.L., Lazzara, E.H., Salas, E. Burke, C.S. Estock, J.L., Orvis, L. K. \& Conkey, C. (2009). Relationships Between Game Attributes and Learning Outcomes: Review and Research Proposals. Simulation \& Gaming, 40(2), 217-266

Zichermann, G., \& Cunningham, C. (2011). Gamification by Design: Implementing Game Mechanics in Web and Mobile Apps. Sebastopol: O'Reilly Media. 